Source: Daraei M, Zolfigol MA, Derakhshan-Panah F, Shiri M, Kruger HG, Mokhlesi M: Synthesis of tetrahydropyridines by one-pot multicomponent reaction using nano-sphere silica sulfuric acid. J Iran Chem Soc 2015, 12(5):855-861. $\underline{10.1007 / s 13738-014-0548-x}$

\title{
Synthesis of tetrahydropyridines by one-pot multicomponent reaction using nano-sphere silica sulfuric acid
}

\author{
Masoume Daraei ${ }^{1}$, Mohammad Ali Zolfigol ${ }^{1}$, Fateme Derakhshan-Panah ${ }^{1}$, Morteza Shiri ${ }^{2}$, Hendrik G. \\ Kruger $^{3}$, Mohammad Mokhlesi ${ }^{4}$
}

1 Faculty of Chemistry, Bu-Ali Sina University, P.O. Box 6517838683, Hamedan, Iran.

2 Department of Chemistry Faculty of Science, Alzahra University, 1993893973 Vanak, Tehran, Iran.

3 Catalysis and Peptide Research Unit, School of Health Sciences, University of KwaZulu-Natal, Durban 4001, South Africa.

4 Pishro Parsian Company, 11th floor, No. 1917, Amir Bldg, above the four way the Jalal alahmad, North Kargar Ave, 1439713134 Tehran, Iran e-mail: mmokhlesi313@gmail.com.

\begin{abstract}
A highly efficient protocol for the synthesis of 1,2,3,4-tetrahydropyridines in the presence of nano-sphere silica sulfuric acid (NS-SSA) was used for good yields by one-pot multicomponent reaction (MCRs). The reagent nano-sphere silica sulfuric acid (NS-SSA) has several advantages, such as easy workup, nontoxicity, convenience and high yields of products.
\end{abstract}

Keywords: Tetrahydropyridines, Nano-sphere silica sulfuric acid, Multicomponent reaction. 